\title{
COVID-19 biomarkers for severity mapped to polycystic ovary syndrome
}

\author{
Abu Saleh Md Moin ${ }^{1}$, Thozhukat Sathyapalan², Stephen L. Atkin ${ }^{3 \dagger}$ and Alexandra E. Butler ${ }^{1 * \dagger}$ (D)
}

To the Editor,

Large scale multi-omics analysis has identified significant differences in the biomarkers between COVID-19 disease and control subjects [1]. These protein panels target biological processes involved in vessel damage, platelet degranulation, the coagulation cascade and the acute phase response [1], with greater protein changes dependent on the COVID-19 severity. However, it is observed that in metabolic conditions such as polycystic ovary syndrome expressed proteins differ compared to control women [2] and PCOS patients have increased platelet aggregation and decreased plasma fibrinolytic activity, resulting in a prothrombotic propensity $[3,4]$, with elevated coagulation markers [5]. Therefore, any biomarkers reflecting COVID-19 disease and its severity would necessarily have to be independent of differentiallyexpressed proteins relating to other conditions; therefore, this proteomic analysis was undertaken in women with and without PCOS to compare with the proteomic biomarkers recently described in COVID-19 using shotgun proteomics followed by parallel reaction monitoring [1].

146 PCOS and 97 control women who presented sequentially to the Department of Endocrinology, Hull and East Yorkshire Hospitals NHS Trust were recruited to the local PCOS biobank (ISRCTN70196169) [2]. PCOS diagnosis was based on all three Rotterdam consensus

\footnotetext{
*Correspondence: aeb91011@gmail.com; abutler@hbku.edu.qa

†'Stephen L. Atkin and Alexandra E. Butler are joint senior authors.

${ }^{1}$ Diabetes Research Center (DRC), Qatar Biomedical Research Institute (QBRI), Hamad Bin Khalifa University (HBKU), Qatar Foundation (QF), PO Box 34110, Doha, Qatar

Full list of author information is available at the end of the article
}

diagnostic criteria. Proteins that were identified as being altered in COVID-19 disease for vessel damage (16 proteins), platelet degranulation (11 proteins), coagulation cascade (24 proteins) and acute phase response ( 9 proteins), shown in Table 1, were determined by Slow Off-rate Modified Aptamer (SOMA)-scan plasma protein measurement [6]. Statistics were performed using Graphpad Prism 8.0.

As reported previously [2], cohorts were age-matched, but PCOS women had increased insulin resistance, androgens and CRP $(\mathrm{p}<0.001)$ : systolic and diastolic blood pressure, and waist circumference were higher $(\mathrm{p}<0.05)$.

For the 60 protein biomarkers described previously, 21 were found to differ in PCOS: for vessel damage 7 of 16 proteins differed, for platelet degranulation 2 of 11 proteins differed, for the coagulation cascade 9 of 24 proteins differed and for the acute phase response 3 of 9 proteins differed (Table 1).

These data show that altered protein expression relative to controls may occur in other conditions such as PCOS, and that COVID-19 biomarker changes found between respiratory patients with and without COVID-19 require validation before they can be confirmed to be related to COVID-19 disease and its severity. It is of concern that a number of significant protein biomarkers described for COVID-19 patients and its severity were also found in PCOS, spanning the biological processes involved in vessel damage, platelet degranulation, the coagulation cascade and the acute phase response, perhaps indicating why these patients may be at risk of more severe COVID19 disease [7].

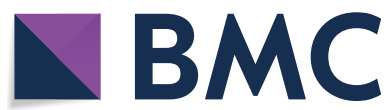

(c) The Author(s) 2020, corrected publication 2021. This article is licensed under a Creative Commons Attribution 4.0 International License, which permits use, sharing, adaptation, distribution and reproduction in any medium or format, as long as you give appropriate credit to the original author(s) and the source, provide a link to the Creative Commons licence, and indicate if changes were made. The images or other third party material in this article are included in the article's Creative Commons licence, unless indicated otherwise in a credit line to the material. If material is not included in the article's Creative Commons licence and your intended use is not permitted by statutory regulation or exceeds the permitted use, you will need to obtain permission directly from the copyright holder. To view a copy of this licence, visit http://creativecommons.org/licenses/by/4.0/. The Creative Commons Public Domain Dedication waiver (http://creativeco mmons.org/publicdomain/zero/1.0/) applies to the data made available in this article, unless otherwise stated in a credit line to the data. 
Table 1 Proteins identified as being altered in COVID-19 disease categorized according to biological processes: vessel damage (16 proteins), platelet degranulation (11 proteins), coagulation cascade (24 proteins) and acute phase response (9 proteins) in PCOS and control women

\begin{tabular}{|c|c|c|c|c|c|}
\hline Target full name & Target & UniProt & Entrez gene ID & Entrez gene symbol & $\begin{array}{l}\text { T-test PCOS } \\
\text { vs control }\end{array}$ \\
\hline \multicolumn{6}{|l|}{ Vessel damage } \\
\hline Angiotensinogen & Angiotensinogen & P01019 & 183 & AGT & 0.0240 \\
\hline Angiopoietin-1 & Angiopoietin-1 & Q15389 & 284 & ANGPT1 & 0.0760 \\
\hline Angiogenin & Angiogenin & P03950 & 283 & ANG & 0.2540 \\
\hline $\begin{array}{l}\text { EGF-containing fibulin-like extra- } \\
\text { cellular matrix protein } 1\end{array}$ & FBLN3 & Q12805 & 2202 & EFEMP1 & 0.4220 \\
\hline Gelsolin & Gelsolin & P06396 & 2934 & GSN & 0.0002 \\
\hline Hemopexin & Hemopexin & P02790 & 3263 & $\mathrm{HPX}$ & 0.4390 \\
\hline $\begin{array}{l}\text { Inter-alpha-trypsin inhibitor heavy } \\
\text { chain } \mathrm{H} 4\end{array}$ & ITI heavy chain $\mathrm{H} 4$ & Q14624 & 3700 & $\mathrm{ITIH} 4$ & 0.5360 \\
\hline Lumican & Lumican & P51884 & 4060 & LUM & 0.3790 \\
\hline Nidogen-1 & Nidogen & P14543 & 4811 & NID1 & 0.9600 \\
\hline Neuropilin-1 & NRP1 & 014786 & 8829 & NRP1 & 0.0090 \\
\hline Periostin & Periostin & Q15063 & 10631 & POSTN & 0.0490 \\
\hline $\begin{array}{l}\text { Ras-related C3 botulinum toxin } \\
\text { substrate } 1\end{array}$ & RAC1 & P63000 & 5879 & RAC1 & 0.5850 \\
\hline Kallistatin & Kallistatin & P29622 & 5267 & SERPINA4 & 0.0006 \\
\hline Pigment epithelium-derived factor & PEDF & P36955 & 5176 & SERPINF1 & 0.00003 \\
\hline $\begin{array}{l}\text { Transforming growth factor-beta- } \\
\text { induced protein ig-h3 }\end{array}$ & $\mathrm{BGH} 3$ & Q15582 & 7045 & TGFBI & 0.9890 \\
\hline Tenascin & Tenascin & P24821 & 3371 & TNC & 0.0020 \\
\hline Vitronectin & Vitronectin & P04004 & 7448 & VTN & 0.3930 \\
\hline \multicolumn{6}{|l|}{ Platelet degranulation } \\
\hline Alpha-2-macroglobulin & a2-Macroglobulin & P01023 & 2 & $\mathrm{~A} 2 \mathrm{M}$ & 0.2660 \\
\hline Clusterin & Clusterin & P10909 & 1191 & CLU & 0.6900 \\
\hline Fibronectin & Fibronectin & P02751 & 2335 & FN1 & 0.0098 \\
\hline $\begin{array}{l}\text { Platelet glycoprotein Ib alpha } \\
\text { chain }\end{array}$ & GP1BA & P07359 & 2811 & GP1BA & 0.1560 \\
\hline Histidine-rich glycoprotein & HRG & P04196 & 3273 & HRG & 0.5890 \\
\hline Integrin alpha-IIb: beta-3 complex & gpllbllla & P08514 P05106 & 36743690 & ITGA2B ITGB3 & 0.8370 \\
\hline Neutrophil-activating peptide 2 & NAP-2 & P02775 & 5473 & PPBP & 0.2740 \\
\hline Plasma serine protease inhibitor & $\mathrm{PCl}$ & P05154 & 5104 & SERPINA5 & 0.0380 \\
\hline Corticosteroid-binding globulin & CBG & P08185 & 866 & SERPINA6 & 0.1790 \\
\hline Thyroxine-binding globulin & Thyroxine-Binding Globulin & P05543 & 6906 & SERPINA7 & 0.5730 \\
\hline Transgelin-2 & Transgelin-2 & P37802 & 8407 & TAGLN2 & 0.8690 \\
\hline von Willebrand factor & vWF & P04275 & 7450 & VWF & 0.0770 \\
\hline \multicolumn{6}{|l|}{ Coagulation cascade } \\
\hline Carboxypeptidase B2 & TAFI & Q96IY4 & 1361 & CPB2 & 0.0170 \\
\hline Prothrombin & Prothrombin & P00734 & 2147 & F2 & 0.0920 \\
\hline Coagulation Factor $\vee$ & Coagulation Factor V & P12259 & 2153 & F5 & 0.3020 \\
\hline Coagulation factor VII & Coagulation Factor VII & P08709 & 2155 & F7 & 0.2520 \\
\hline Coagulation factor IX & Coagulation Factor IX & P00740 & 2158 & F9 & $<0.00001$ \\
\hline Coagulation factor Xa & Coagulation Factor Xa & P00742 & 2159 & F10 & 0.1620 \\
\hline Coagulation Factor XI & Coagulation Factor XI & P03951 & 2160 & F11 & 0.5190 \\
\hline Fibrinogen & Fibrinogen & P02671 P02675 P02679 & 224322442266 & FGA FGB FGG & 0.0126 \\
\hline D-dimer & D-dimer & P02671 P02675 P02679 & 224322442266 & FGA FGB FGG & 0.00002 \\
\hline Fibrinogen gamma chain & Fibrinogen g-chain dimer & P02679 & 2266 & FGG & 0.00002 \\
\hline
\end{tabular}


Table 1 (continued)

\begin{tabular}{|c|c|c|c|c|c|}
\hline Target full name & Target & UniProt & Entrez gene ID & Entrez gene symbol & $\begin{array}{l}\text { T-test PCOS } \\
\text { vs control }\end{array}$ \\
\hline $\begin{array}{l}\text { Hepatocyte growth factor activa- } \\
\text { tor }\end{array}$ & HGFA & Q04756 & 3083 & HGFAC & 0.0830 \\
\hline Plasma kallikrein & Prekallikrein & P03952 & 3818 & KLKB1 & 0.0015 \\
\hline Kininogen-1 & Kininogen, HMW & P01042 & 3827 & KNG1 & 0.7970 \\
\hline Plasminogen & Plasminogen & P00747 & 5340 & PLG & 0.6740 \\
\hline Vitamin K-dependent protein S & Protein S & P07225 & 5627 & PROS1 & 0.00003 \\
\hline Vitamin K-dependent protein C & Protein C & P04070 & 5624 & PROC & 0.5110 \\
\hline Alpha-1-antitrypsin & a1-Antitrypsin & P01009 & 5265 & SERPINA1 & 0.1500 \\
\hline $\begin{array}{l}\text { Protein Z-dependent protease } \\
\text { inhibitor }\end{array}$ & protein Z inhibitor & Q9UK55 & 51156 & SERPINA10 & 0.1720 \\
\hline Antithrombin-III & Antithrombin III & P01008 & 462 & SERPINC1 & 0.0003 \\
\hline Heparin cofactor 2 & Heparin cofactor II & P05546 & 3053 & SERPIND1 & 0.00003 \\
\hline Plasminogen activator inhibitor 1 & PAl-1 & P05121 & 5054 & SERPINE1 & 0.1680 \\
\hline Alpha-2-antiplasmin & a2-Antiplasmin & P08697 & 5345 & SERPINF2 & \\
\hline \multicolumn{6}{|l|}{ Acute phase response } \\
\hline Serum albumin & Albumin & P02768 & 213 & ALB & 0.0660 \\
\hline Macrophage mannose receptor 1 & Macrophage mannose receptor & P22897 & 4360 & $\mathrm{MRC1}$ & 0.0020 \\
\hline $\begin{array}{l}\text { Hepatocyte growth factor-like } \\
\text { protein }\end{array}$ & MSP & P26927 & 4485 & MST1 & 0.7340 \\
\hline Protein S100-A9 & calgranulin B & P06702 & 6280 & S100A9 & 0.0156 \\
\hline Serum amyloid A-1 protein & SAA & 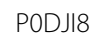 & 6288 & SAA1 & 0.5095 \\
\hline Alpha-1-antichymotrypsin & a1-Antichymotrypsin & P01011 & 12 & SERPINA3 & 0.2672 \\
\hline Superoxide dismutase [Cu-Zn] & SOD & P00441 & 6647 & SOD1 & 0.9448 \\
\hline Serotransferrin & Transferrin & P02787 & 7018 & TF & 0.0185 \\
\hline Transketolase & Transketolase & P29401 & 7086 & TKT & 0.8309 \\
\hline
\end{tabular}

Limitations of the study include utilizing a different method of proteomic analysis compared to others that may not be directly comparable [1].

In conclusion, 21 of 60 protein biomarkers reported in respiratory patients with COVID-19 were found to differ between women with and without PCOS, showing the necessity for validation of such biomarkers, and suggesting that more severe COVID-19 disease may occur in PCOS.

\section{Acknowledgements}

None.

\section{Authors' contributions}

ASMM and AEB analyzed the data and wrote the manuscript. TS supervised clinical studies and edited the manuscript. SLA contributed to study design, data interpretation and the writing of the manuscript. AEB is the guarantor of this work. All authors read and approved the final manuscript.

\section{Funding}

No funding was received to perform this study.

\section{Availability of data and materials}

All the data for this study will be made available upon reasonable request to the corresponding author.

\section{Ethics approval and consent to participate}

The Newcastle \& North Tyneside Ethics committee approved this study that was conducted according to the Declaration of Helsinki. All study participants signed an informed consent form prior to participation.

\section{Consent for publication}

All authors gave their consent for publication.

\section{Competing interests}

No authors have any conflict of interest or competing interests to declare.

\section{Author details}

${ }^{1}$ Diabetes Research Center (DRC), Qatar Biomedical Research Institute (QBRI), Hamad Bin Khalifa University (HBKU), Qatar Foundation (QF), PO Box 34110 , Doha, Qatar. ${ }^{2}$ Academic Endocrinology, Diabetes and Metabolism, Hull York Medical School, Hull, UK. ${ }^{3}$ Royal College of Surgeons in Ireland Bahrain, Adliya, Kingdom of Bahrain.

Received: 14 November 2020 Accepted: 4 December 2020

Published online: 22 December 2020

\section{References}

1. Overmyer KA, Shishkova E, Miller IJ, Balnis J, Bernstein MN, Peters-Clarke TM, et al. Large-Scale Multi-omic Analysis of COVID-19 Severity. Cell Syst. 2020. https://doi.org/10.1016/j.cels.2020.10.003. 
2. Moin ASM, Sathyapalan T, Atkin SL, Butler AE. Renin-Angiotensin System Overactivation in Polycystic Ovary Syndrome, a Risk for SARS-CoV-2 Infection? Metabol Open. 2020;7:100052.

3. Mak W, Dokras A. Polycystic ovarian syndrome and the risk of cardiovascular disease and thrombosis. Semin Thromb Hemost. 2009;35(7):613-20.

4. Yildiz BO, Haznedaroğlu IC, Kirazli S, Bayraktar M. Global fibrinolytic capacity is decreased in polycystic ovary syndrome, suggesting a prothrombotic state. J Clin Endocrinol Metabol. 2002;87(8):3871-5.

5. Oral B, Mermi B, Dilek M, Alanoğlu G, Sütçü R. Thrombin activatable fibrinolysis inhibitor and other hemostatic parameters in patients with polycystic ovary syndrome. Gynecol Endocrinol. 2009;25(2):110-6.
6. Kahal H, Halama A, Aburima A, Bhagwat AM, Butler AE, Grauman J, et al. Effect of induced hypoglycemia on inflammation and oxidative stress in type 2 diabetes and control subjects. Sci Rep. 2020;10(1):4750.

7. Juusela A, Nazir M, Gimovsky M. Two cases of coronavirus 2019-related cardiomyopathy in pregnancy. Am J Obstet Gynecol MFM. 2020;2(2):100113.

\section{Publisher's Note}

Springer Nature remains neutral with regard to jurisdictional claims in published maps and institutional affiliations.
Ready to submit your research? Choose BMC and benefit from:

- fast, convenient online submission

- thorough peer review by experienced researchers in your field

- rapid publication on acceptance

- support for research data, including large and complex data types

- gold Open Access which fosters wider collaboration and increased citations

- maximum visibility for your research: over $100 \mathrm{M}$ website views per year

At BMC, research is always in progress.

Learn more biomedcentral.com/submissions 